\title{
BOUNDS ON THE NUMBER OF REAL SOLUTIONS TO POLYNOMIAL EQUATIONS
}

\author{
DANIEL J. BATES, FRÉDÉRIC BIHAN, AND FRANK SOTTILE
}

\begin{abstract}
We use Gale duality for polynomial complete intersections and adapt the proof of the fewnomial bound for positive solutions to obtain the bound

$$
\frac{e^{4}+3}{4} 2^{\left(\begin{array}{c}
k \\
2
\end{array}\right) n^{k}}
$$

for the number of non-zero real solutions to a system of $n$ polynomials in $n$ variables having $n+k+1$ monomials whose exponent vectors generate a subgroup of $\mathbb{Z}^{n}$ of odd index. This bound exceeds the bound for positive solutions only by the constant factor $\left(e^{4}+3\right) /\left(e^{2}+3\right)$ and it is asymptotically sharp for $k$ fixed and $n$ large.
\end{abstract}

\section{INTRODUCTION}

In [3], the sharp bound of $2 n+1$ was obtained for the number of non-zero real solutions to a system of $n$ polynomial equations in $n$ variables having $n+2$ monomials whose exponents affinely span the lattice $\mathbb{Z}^{n}$. In [4, the sharp bound of $n+1$ was given for the solutions in the positive orthant to such a system of equations. This last bound was generalized in [6], which showed that the number of solutions in the positive orthant to a system of $n$ polynomial equations in $n$ variables having $n+k+1$ monomials was less than

$$
\frac{e^{2}+3}{4} 2^{\left(\begin{array}{c}
k \\
2
\end{array}\right)} n^{k}
$$

which is asymptotically sharp for $k$ fixed and $n$ large [5. This dramatically improved

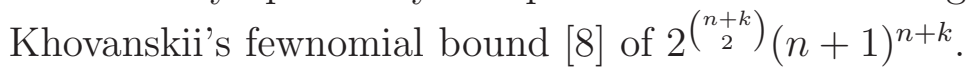

We generalize this to a bound for all non-zero real solutions. Under the assumption that the exponent vectors $\mathcal{W}$ span a subgroup of $\mathbb{Z}^{n}$ of odd index, we show that the number of non-zero real solutions to a system of polynomials with support $\mathcal{W}$ is less than

$$
\frac{e^{4}+3}{4} 2^{\left(\begin{array}{c}
k \\
2
\end{array}\right)} n^{k}
$$

The novelty of this bound is that it exceeds the bound for solutions in the positive orthant by a fixed constant factor $\left(e^{4}+3\right) /\left(e^{2}+3\right)$, rather than by a factor of $2^{n}$, which is the number of orthants. By the construction in [5], it is asymptotically sharp for $k$ fixed and $n$ large.

2000 Mathematics Subject Classification. 14M25, 14P25, 52C35.

Key words and phrases. sparse polynomial system, hyperplane arrangement, fewnomial.

Bates and Sottile supported by the Institute for Mathematics and its Applications.

Sottile supported by the NSF CAREER grant DMS-0538734. 
We follow the outline of [6] — we use Gale duality for real complete intersections [7] and then bound the number of solutions to the dual system of master functions. The key idea is that using all chambers of a complement of an arrangement of hyperplanes in $\mathbb{R P}^{k}$, rather than one chamber as in [6], does not increase our estimate on the numbers of solutions too much. This was discovered while implementing a numerical continuation algorithm for computing the positive solutions to a system of polynomials [1]. That algorithm finds all real solutions without computing complex solutions and is based on [6]. Its complexity depends on (1), and not on the number of complex solutions.

We state our main theorem in Section 1 and then use Gale duality to reduce it to a statement about systems of master functions, which we prove in Section 2.

\section{Gale DUAlity FOR Systems of SPARSE POLYNOMials}

Let $\mathcal{W}=\left\{w_{0}=0, w_{1}, \ldots, w_{n+k}\right\} \subset \mathbb{Z}^{n}$ be a collection of $n+k+1$ integer vectors $(|\mathcal{W}|=$ $n+k+1$ ), which correspond to monomials in variables $x_{1}, \ldots, x_{n}$. A (Laurent) polynomial $f$ with support $\mathcal{W}$ is a real linear combination of monomials with exponents from $\mathcal{W}$,

$$
f\left(x_{1}, \ldots, x_{n}\right)=\sum_{i=0}^{n+k} c_{i} x^{w_{i}} \quad \text { with } c_{i} \in \mathbb{R} .
$$

A system with support $\mathcal{W}$ is a system of polynomial equations

$$
f_{1}\left(x_{1}, \ldots, x_{n}\right)=f_{2}\left(x_{1}, \ldots, x_{n}\right)=\cdots=f_{n}\left(x_{1}, \ldots, x_{n}\right)=0,
$$

where each polynomial $f_{i}$ has support $\mathcal{W}$. Since multiplying every polynomial in (3) by a monomial $x^{\alpha}$ does not change the solution set but translates $\mathcal{W}$ by the vector $\alpha$, we see that it was no loss of generality to assume that $0 \in \mathcal{W}$.

The system (3) has infinitely many solutions if $\mathcal{W}$ does not span $\mathbb{R}^{n}$. We say that $\mathcal{W}$ spans $\mathbb{Z}^{n} \bmod 2$ if the $\mathbb{Z}$-linear span of $\mathcal{W}$ is a subgroup of $\mathbb{Z}^{n}$ of odd index.

Theorem 1. Suppose that $\mathcal{W}$ spans $\mathbb{Z}^{n} \bmod 2$ and $|\mathcal{W}|=n+k+1$. Then there are fewer than (1) non-degenerate non-zero real solutions to a sparse system (3) with support $\mathcal{W}$.

The importance of this bound for the number of real solutions is that it has a completely different character than Kouchnirenko's bound for the number of complex solutions.

Proposition 2 (Kouchnirenko [2]). The number of non-degenerate solutions in $\left(\mathbb{C}^{\times}\right)^{n}$ to a system (3) with support $\mathcal{W}$ is no more than $n ! \operatorname{vol}(\operatorname{conv}(\mathcal{W}))$.

Here, $\operatorname{vol}(\operatorname{conv}(\mathcal{W}))$ is the Euclidean volume of the convex hull of $\mathcal{W}$.

Perturbing coefficients of the polynomials in (3) so that it is a complete intersection in $\left(\mathbb{C}^{\times}\right)^{n}$ can only increase the number of non-degenerate solutions. Thus it suffices to prove Theorem 1 under this assumption. Such a complete intersection is equivalent to a complete intersection of master functions in a hyperplane complement [7].

Let $\mathbb{R}^{n+k}$ have coordinates $z_{1}, \ldots, z_{n+k}$. A polynomial (2) with support $\mathcal{W}$ is the pullback $\Phi_{\mathcal{W}}^{*}(\Lambda)$ of the linear form $\Lambda:=c_{0}+c_{1} z_{1}+\cdots+c_{n+k} z_{n+k}$ along the map

$$
\Phi_{\mathcal{W}}:\left(\mathbb{R}^{\times}\right)^{n} \ni x \longmapsto\left(x^{w_{i}} \mid i=1, \ldots, n+k\right) \in \mathbb{R}^{n+k} .
$$


If we let $\Lambda_{1}, \ldots, \Lambda_{n}$ be the linear polynomials which pull back to the polynomials in the system (3), then they cut out an affine subspace $L$ of $\mathbb{R}^{n+k}$ of dimension $k$.

Let $\left\{p_{i} \mid i=1, \ldots, n+k\right\}$ be linear polynomials on $\mathbb{R}^{k}$ which induce an isomorphism

$$
\Psi_{p}: \mathbb{R}^{k} \ni y \longmapsto\left(p_{1}(y), \ldots, p_{n+k}(y)\right) \in L \subset \mathbb{R}^{n+k} .
$$

Let $\mathcal{A} \subset \mathbb{R}^{k}$ be the arrangement of hyperplanes defined by the vanishing of the $p_{i}(y)$. This is the pullback along $\Psi_{p}$ of the coordinate hyperplanes of $\mathbb{R}^{n+k}$.

The image $\Phi_{\mathcal{W}}\left(\left(\mathbb{R}^{\times}\right)^{n}\right)$ inside of the torus $\left(\mathbb{R}^{\times}\right)^{n+k}$ has equations

$$
z^{\beta_{1}}=z^{\beta_{2}}=\cdots=z^{\beta_{k}}=1 \text {, }
$$

where the weights $\left\{\beta_{1}, \ldots, \beta_{k}\right\}$ form a basis for the $\mathbb{Z}$-submodule of $\mathbb{Z}^{n+k}$ of linear relations among the vectors $\mathcal{W}$. To these data, we associate a system of master functions on the complement $M_{\mathcal{A}}$ of the arrangement $\mathcal{A}$ of $\mathbb{R}^{k}$,

$$
p(y)^{\beta_{1}}=p(y)^{\beta_{2}}=\cdots=p(y)^{\beta_{k}}=1 .
$$

Here, if $\beta=\left(b_{1}, \ldots, b_{n+k}\right)$ then $p^{\beta}:=p_{1}(y)^{b_{1}} \cdots p_{n+k}(y)^{b_{n+k}}$.

A basic result of [7, Section 4] is that if $\mathcal{W}$ spans $\mathbb{Z}^{n}$ modulo 2 and either of the systems (3) or (41) is a complete intersection, then the other is a complete intersection and the maps $\Phi_{\mathcal{W}}$ and $\Psi_{p}$ induce isomorphisms between the two solution sets, as analytic subschemes of $\left(\mathbb{R}^{\times}\right)^{n}$ and $M_{\mathcal{A}}$. Since we assumed that the system (3) is generic, these hypotheses hold and the arrangement is essential in that the polynomials $p_{i}$ span the space of all linear polynomials on $\mathbb{R}^{k}$.

Theorem 3. A system (4) of master functions in the complement of an essential arrangement of $n+k$ hyperplanes in $\mathbb{R}^{k}$ has at most (1) non degenerate real solutions.

We actually prove a bound for a more general system than (4), namely for

$$
p(z)^{2 \beta_{1}}=p(z)^{2 \beta_{2}}=\cdots=p(z)^{2 \beta_{k}}=1 .
$$

We write this more general system as

$$
|p(z)|^{\beta_{1}}=|p(z)|^{\beta_{2}}=\cdots=|p(z)|^{\beta_{k}}=1 .
$$

In a system of this form we may have real number weights $\beta_{i} \in \mathbb{R}^{n+k}$.

Theorem 4. A system of the form (15) with real weights in the complement of an essential arrangement of $n+k$ hyperplanes in $\mathbb{R}^{k}$ has at most (11) non degenerate real solutions.

\section{Proof of Theorem 4}

We follow [6] with minor, but important, modifications. Perturbing the polynomials $p_{i}(y)$ and the weights $\beta_{j}$ will not decrease the number of non-degenerate real solutions in $M_{\mathcal{A}}$. This enables us to make the following assumptions.

The arrangement $\mathcal{A}^{+} \subset \mathbb{R P}^{k}$, where we add the hyperplane at infinity, is generic in that every $j$ hyperplanes of $\mathcal{A}^{+}$meet in a $(k-j)$ dimensional linear subspace, called a codimension $j$ face of $\mathcal{A}$. If $B$ is the matrix whose columns are the weights $\beta_{1}, \ldots, \beta_{k}$, then the entries of $B$ are rational numbers and no minor of $B$ vanishes. This last technical 
condition as well as the freedom to further perturb the $\beta_{j}$ and the $p_{i}$ are necessary for the results in [6, Section 3] upon which we rely.

For functions $f_{1}, \ldots, f_{j}$ on $M_{\mathcal{A}}$, let $V\left(f_{1}, \ldots, f_{j}\right)$ be the subvariety they define. Suppose that $\beta_{j}=\left(b_{1, j}, \ldots, b_{n+k, j}\right)$. For each $j=1, \ldots, k$, define

$$
\psi_{j}(y):=\sum_{i=1}^{n+k} b_{i, j} \log \left|p_{i}(y)\right| .
$$

Then (5) is equivalent to $\psi_{1}(y)=\cdots=\psi_{k}(y)=0$. Define $\Gamma_{k}, \Gamma_{k-1}, \ldots, \Gamma_{1}$ by

$$
\Gamma_{j}:=\operatorname{Jac}\left(\psi_{1}, \ldots, \psi_{j}, \Gamma_{j+1}, \ldots, \Gamma_{k}\right),
$$

the Jacobian determinant of $\psi_{1}, \ldots, \psi_{j}, \Gamma_{j+1}, \ldots, \Gamma_{k}$. Set

$$
C_{j}:=V\left(\psi_{1}, \ldots, \psi_{j-1}, \Gamma_{j+1}, \ldots, \Gamma_{k}\right)
$$

Let $b(C)$ be the number of unbounded components of a curve $C \subset M_{\mathcal{A}}$. We have the estimate from [6], which is a consequence of the Khovanskii-Rolle Theorem,

$$
\left|V\left(\psi_{1}, \ldots, \psi_{k}\right)\right| \leq b\left(C_{k}\right)+\cdots+b\left(C_{1}\right)+\left|V\left(\Gamma_{1}, \ldots, \Gamma_{k}\right)\right| .
$$

Here, $|S|$ is the cardinalty of the set $S$. We estimate these quantities.

\section{Lemma 5.}

(1) $\left|V\left(\Gamma_{1}, \ldots, \Gamma_{k}\right)\right| \leq 2^{\left(\begin{array}{c}k \\ 2\end{array}\right)} n^{k}$.

(2) $C_{j}$ is a smooth curve and

$$
b\left(C_{j}\right) \leq \frac{1}{2} 2^{\left(\begin{array}{c}
k-j \\
2
\end{array}\right)} n^{n-k}\left(\begin{array}{c}
n+k+1 \\
j
\end{array}\right) \cdot 2^{j} \leq 2^{\left(\begin{array}{c}
k \\
2
\end{array}\right) n^{k} \cdot \frac{2^{2 j-1}}{j !} .}
$$

Proof of Theorem 4. By (6) and Lemma 5, we have

$$
\left|V\left(\psi_{1}, \ldots, \psi_{k}\right)\right| \leq 2^{\left(\begin{array}{c}
k \\
2
\end{array}\right)} n^{k}\left(1+\frac{1}{4} \sum_{j=1}^{k} \frac{4^{j}}{j !}\right)<2^{\left(\begin{array}{c}
k \\
2
\end{array}\right)} n^{k} \cdot \frac{e^{4}+3}{4} .
$$

Proof of Lemma 5. The bound (1) is from Lemma 3.4 of 6]. Statements analogous to (2) for $\widetilde{C}_{j}$, the restriction of $C_{j}$ to a single chamber (connected component) of $M_{\mathcal{A}}$ were established in Lemma 3.4 and the proof of Lemma 3.5 in [6]:

$$
b\left(\widetilde{C}_{j}\right) \leq \frac{1}{2} 2^{\left(\begin{array}{c}
k-j \\
2
\end{array}\right)} n^{n-k}\left(\begin{array}{c}
n+k+1 \\
j
\end{array}\right) \leq 2^{\left(\begin{array}{c}
k \\
2
\end{array}\right)} n^{k} \cdot \frac{2^{j-1}}{j !} .
$$

The bound we claim for $b\left(C_{j}\right)$ has an extra factor of $2^{j}$. A priori we would expect to multiply this bound (77) by the number of chambers of $M_{\mathcal{A}}$ to obtain a bound for $b\left(C_{j}\right)$, but the correct factor is only $2^{j}$.

We work in $\mathbb{R P}^{k}$ and use the extended hyperplane arrangement $\mathcal{A}^{+}$, as we will need points in the closure of $C_{j}$ in $\mathbb{R P}^{k}$. The first inequality in (7) for $b\left(\widetilde{C}_{j}\right)$ arises as each 
unbounded component of $\widetilde{C}_{j}$ meets $\mathcal{A}^{+}$in two distinct points (this accounts for the factor $\left.\frac{1}{2}\right)$ which are points of codimension $j$ faces where the polynomials

$$
F_{i}(y):=\Gamma_{k-i}(y) \cdot\left(\prod_{i=1}^{n+k} p_{i}(y)\right)^{2^{i}}
$$

for $i=0, \ldots, k-j-1$ vanish. (By Lemma 3.4(1) of [6], $F_{i}$ is a polynomial of degree $2^{i} n$.) The genericity of the weights and the linear polynomials $p_{i}(y)$ imply that these points will lie on faces of codimension $j$ but not of higher codimension. The factor $2^{\left({ }^{k-j}\right)} n^{n-k}$ is the Bézout number of the system $F_{0}=\cdots=F_{k-j-1}$ on a given codimension $j$ plane, and there are exactly $\left(\begin{array}{c}n+k+1 \\ j\end{array}\right)$ codimension $j$ faces of $\mathcal{A}^{+}$.

At each of these points, $C_{j}$ will have one branch in each chamber of $M_{\mathcal{A}}$ incident on that point. Since the hyperplane arrangement $\mathcal{A}^{+}$is generic there will be exactly $2^{j}$ such chambers.

\section{REFERENCES}

[1] D.J. Bates and F. Sottile, Khovanskii-Rolle continuation for real solutions, 2007, in preparation.

[2] D. Bernstein, A. Kouchnirenko, and A. Khovanskii, Newton polytopes, Usp. Math. Nauk. 31 (1976), no. 3, 201-202, (in Russian).

[3] B. Bertrand, F. Bihan, and F. Sottile, Polynomial systems with few real zeroes, Math. Z. 253 (2006), no. 2, 361-385.

[4] F. Bihan, Polynomial systems supported on circuits and dessins d'enfants, Journal of the London Mathematical Society 75 (2007), no. 1, 116-132.

[5] F. Bihan, J.M. Rojas, and F. Sottile, Sharpness of fewnomial bounds and the number of components of a fewnomial hypersurface, 2007, IMA volume on algorithms in algebraic geometry, to appear.

[6] F. Bihan and F. Sottile, New fewnomial upper bounds from Gale dual polynomial systems, 2006, Moscow Mathematical Journal, to appear. math.AG/0609544.

[7] - Gale duality for complete intersections, 2007, arXiv:0706.3745, 10 pages.

[8] A.G. Khovanskii, A class of systems of transcendental equations, Dokl. Akad. Nauk. SSSR 255 (1980), no. 4, 804-807.

Institute for Mathematics And its Applichtions, University of Minnesota, 114 Lind Hall, 207 Church Street S.E., Minneapolis, MN 55455-0436, USA

E-mail address: dbates1@nd.edu

$U R L:$ http: //www.nd.edu/ dbates1/

Laboratoire de Mathématiques, Université de Savoie, 73376 Le Bourget-Du-Lac Cedex, FRANCE

E-mail address: Frederic.Bihan@univ-savoie.fr

URL: http://www. lama.univ-savoie.fr/ bihan/

Department of Mathematics, Texas A\&M University, College Station, Texas 77843, USA

E-mail address: sottile@math.tamu.edu

$U R L:$ http: //www . math.tamu.edu/ sottile/ 